\title{
Phase behavior of (polyacrylamides + water) solutions: concentration, pressure and isotope effects
}

\author{
R. Gomes de Azevedo ${ }^{\text {a }}$, L.P.N. Rebelo ${ }^{\text {a, } *}$, A.M. Ramos ${ }^{\text {a }}$, \\ J. Szydlowski ${ }^{b}$, H.C. de Sousa ${ }^{c}$, J. Klein ${ }^{\mathrm{d}}$ \\ a Instituto de Tecnologia Química e Biológica, ITQB 2, Universidade Nova de Lisboa, Av. República, \\ Apartado 127, 2780-901 Oeiras, Portugal \\ ${ }^{\mathrm{b}}$ Chemistry Department, Warsaw University, Zwirki I Wigury 101, 02-089 Warsaw, Poland

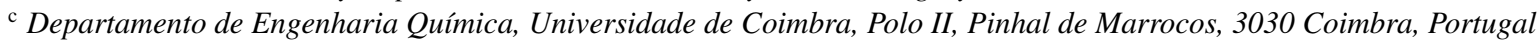 \\ ${ }^{\mathrm{d}}$ Lehrstuhl für Makromolekulare Chemie, Technische Universität Braunschweig, \\ Hans-Sommer-Str. 10, D-38106 Braunschweig, Germany
}

\begin{abstract}
Phase diagrams of poly $(N$-isopropylacrylamide) (PNIPAAM) as well as of hydrophilically-modified copolymers in aqueous solution were determined. A high-accuracy He-Ne Laser scattering technique was used for the detection of operational spinodal (sp) and cloud-point (cp) curves. Polymer concentration was varied from 0.5 to $20 \mathrm{wt} . \%$. In the case of copoly(PNIPAAM/vinylsaccharide) several different chain lengths were considered. Pressure (up to 400 bar) and solvent isotope effects were studied. We predict a closed-loop type phase diagram for the copolymers, which presents an estimated hypercritical point in $\mathrm{H}_{2} \mathrm{O}$ solutions at $M_{\mathrm{w}} \sim 0.5 \times 10^{5}$, although only lower critical solution temperature (LCST) is experimentally accessible. At lower molecular weights, the solutions are always in the one-phase region. (C) 2001 Elsevier Science B.V. All rights reserved.
\end{abstract}

Keywords: Data; Liquid-liquid equilibria; Polyacrylamides; Polymeric aqueous solutions; Pressure effects; Isotope effects

\section{Introduction}

Poly( $N$-isopropylacrylamide), so-called PNIPAAM, is fairly soluble in water at room temperature and atmospheric pressure. Interest from the scientific and industrial community in PNIPAAM, has been growing, especially in the last decade, since it has a large number of potential applications including immobilization of enzymes and protein separation [1,2]. It has probably become the most popular member of a class of polymers that possesses inverse solubility in aqueous solution upon heating, a property which it shares with some other polymers capable of interacting through hydrogen-bonds with water, but

\footnotetext{
* Corresponding author. Tel.: +351-21-4469-441; fax: +351-21-4411-277.

E-mail address: luis.rebelo@itqb.unl.pt (L.P.N. Rebelo).
} 
contrary to the behavior of most polymers in solution. Despite current interest in PNIPAAM, accurate data concerning its phase equilibria in water [1-5] are still scarce, hence this work.

The degree of solubility and the $(P, T, x)$ conditions of its occurrence are a consequence of the interplay between hydrophilic and hydrophobic forces in solution, and experimentally observed phase transition occurs at the lower critical solution temperature (LCST) curve. An easy way of controlling the hydrophilic/hydrophobic balance is to add sugars in distinct proportions. This can be done either in the solution (where the sugar competes with the polymer for dissolution) or in the polymer (copolymerization), or in both. In this work, we have therefore, chosen to study a copolymer [6] as well as PNIPAAM. The copolymer contains about $13-14 \mathrm{~mol} \%$ of a vinylsaccharide (VS) highly hydrophilic, 1-deoxy-1-methacrylamido-D-glucitol, in PNIPAAM. Aqueous solutions of copolymer + water represent thus, a pseudo-ternary mixture of water + PNIPAAM + VS.

The experimental technique for the detection of phase transitions uses the increase of scattered light and the simultaneous decrease of the transmitted one that results from shining the sample with a $\mathrm{He}-\mathrm{Ne}$ Laser beam as the solution undergoes phase separation [7].

Different polymer concentrations and molecular weights were used. Pressure ranges from atmospheric up to 400 bar. With the aim of achieving a better understanding of the nature of the equilibrium involved, the solvent isotope effect in the phase diagrams of the polymer solutions was also investigated.

\section{Experimental}

\subsection{Polymer synthesis and characterization}

The homopolymer PNIPAAM and several copolymers with a VS monomer, more precisely copoly $(N$ isopropylacrylamide/1-deoxy-1-methacrylamide-D-glucitol), were synthesized by radical polymerization.

The reactions were carried out in $5 \mathrm{~cm}^{3}$ magnetically stirred microvials, under nitrogen atmosphere at $25^{\circ} \mathrm{C}$ in an aqueous solution for $24 \mathrm{~h}$, using the redox pair $\left(\mathrm{NH}_{4}\right)_{2} \mathrm{~S}_{2} \mathrm{O}_{8} / \mathrm{Na}_{2} \mathrm{~S}_{2} \mathrm{O}_{5}$ as a radical initiator. The concentration relationship between the oxidative part of the redox couple $\left(\left(\mathrm{NH}_{4}\right)_{2} \mathrm{~S}_{2} \mathrm{O}_{8}\right.$, concentration $\left.2.0 \times 10^{-2} \mathrm{M}\right)$ and the reductive part $\left(\mathrm{Na}_{2} \mathrm{~S}_{2} \mathrm{O}_{5}\right.$, concentration $\left.7.5 \times 10^{-2} \mathrm{M}\right)$ was maintained constant in all experiments. These concentrations correspond to the values reported in the literature as giving rise to the highest yield on the homopolymerization reaction of 1-deoxy-1-methacrylamido-D-glucitol [6].

In order to obtain products with different average molecular weights, copolymerizations were performed using an initial comonomer composition of $10 \mathrm{~mol} \%$ of VS, and different amounts of radical initiatior, namely $0.2,1.0,1.5$ and $2.0 \%$ (mol\% on monomers). Polymers were purified by ultrafiltration and recovered by freeze-drying.

The VS monomer was previously synthesized according to [6], by reacting 1-amino-1-deoxy-D-sorbitol (Aldrich, Missouri, USA) with methacrylic anhydride (Fluka Chemie AG, Buchs, Switzerland). In the homopolymerization reaction of $\mathrm{N}$-isopropylacrylamide (Aldrich, Missouri, USA) $0.6 \mathrm{~mol} \%$ of monomer of radical initiator was used.

The copolymer composition was determined by elemental analysis of $\mathrm{C}, \mathrm{H}$ and $\mathrm{N}$. Capillary viscometry in $0.1 \mathrm{M} \mathrm{Na}_{2} \mathrm{SO}_{4}$ solution at $25^{\circ} \mathrm{C}$ was performed to determine the viscosity limit number. Using static multi-angle Laser light scattering (Dawn DSP Laser Photometer, Wyatt Tech. Co., California, USA) all copolymers have been characterized to obtain the weight average molecular weight, $\bar{M}_{\mathrm{w}}$. Polydispersities, 
Table 1

Polymer characteristics ${ }^{\mathrm{a}}$

\begin{tabular}{llllll}
\hline Sample & Polymer & VS content $(\mathrm{mol} \%)$ & {$[\eta]\left(\mathrm{ml} \mathrm{g}^{-1}\right)$} & $\bar{M}_{\mathrm{w}} \times 10^{-5}$ & $\bar{M}_{\mathrm{w}} / \bar{M}_{\mathrm{n}}$ \\
\hline P1 & PNIPAAM & - & 62.7 & 6.15 & 2.04 \\
CP1 & Copoly(PNIPAAM/VS) & 14.0 & 56.1 & 4.32 & 2.97 \\
CP2 & Copoly(PNIPAAM/VS) & 12.9 & 16.2 & 1.70 & 2.18 \\
CP3 & Copoly(PNIPAAM/VS) & 13.7 & 1.02 & 1.10 & 2.13 \\
CP4 & Copoly(PNIPAAM/VS) & 13.3 & 0.38 & 0.56 & 1.96 \\
\hline
\end{tabular}

${ }^{\text {a }}$ PNIPAAM: poly( $N$-isopropylacrylamide); VS: 1-deoxy-1-methacrylamido-D-glucitol.

$\bar{M}_{\mathrm{w}} / \bar{M}_{\mathrm{n}}$, have been measured by GPC/SEC in a Waters Co., Massachusetts, USA, apparatus at $25^{\circ} \mathrm{C}$ using water as eluent and a Waters Hydrogel Linear column. Using monodisperse pullulan standards (Shodex, Showa Denko, Japan, in the molecular weight range $0.59 \times 10^{4}$ to $78.8 \times 10^{4}$ ) the calibration curve has been generated. The characteristics of polymer samples used in this work are reported in Table 1.

\subsection{Solvents}

$\mathrm{H}_{2} \mathrm{O}$ used in this work was doubly distilled and deionized (Millipore Co., Bedford, USA, equipment) and stored in sealed microvials. $\mathrm{D}_{2} \mathrm{O}$ was purchased from Aldrich with a claimed purity of 99.9 at. $\% \mathrm{D}$, and used as received. In order to avoid background turbidity, in all cases and prior to the injection into the light scattering cells, solutions were filtered from suspended impurities.

\subsection{Equipment and technique}

The He-Ne Laser light scattering apparatus as well as the methodology used for the detection of phase transitions have very recently been described in great detail [7]. In the case of experiments where pressure was raised above 50 bar, a sapphire/stainless steel cell replaced the original glass capillary cell. In this case, the hydraulic fluid is the pure solvent (in this work, water) contacting a sufficiently long (1/16) in. SS tubing filled with the solution, in order to avoid contamination during compression/expansion cycles. Scattered light intensity is captured at very low angle $(2<2 \theta(\mathrm{deg})<4)$ in the outer part of a bifurcated optical cable [8], while transmitted light is captured in the inner portion of this cable. Scattered $\left(I_{\mathrm{sc}}\right)$ and transmitted light $\left(I_{\mathrm{tr}}\right)$ are corrected for density fluctuations, reflections, and multiple scattering effects according to Debye's theory [9]. The cloud-point (cp) is the point on the $1 / I_{\mathrm{sc}, \text { corr }}$ against pressure $(P)$ or temperature $(T)$ least-squares fits where the slope changes abruptly. The operational spinodal (sp) point will be taken at the interception point where $1 / I_{\mathrm{sc}, \mathrm{corr}}=0$. At this point, the intensity of scattered light has an infinite value. A comprehensive discussion of the methodology of these calculations can be found elsewhere [8], cp temperature accuracy is typically $\pm 0.01 \mathrm{~K}$ in the range $240<T(\mathrm{~K})<400$, decreasing to $\pm 1 \mathrm{~K}$ in the case of sp's, which are obtained from a large extrapolation $[8,10]$ to the point where the inverse of the scattered light intensity reaches a null value. As for pressure, accuracy is \pm 0.1 bar up to 50 bar, decreasing to \pm 1 bar in the range $50<P$ (bar) $<600$. Solutions were made up gravimetrically, and left stirring for several hours (sometimes for several days) in sealed vials before being injected to the measuring cell. 


\section{Results and discussion}

Phase diagrams of polymer solutions are usually represented as two-dimensional cross-sections of a multi-dimensional space that embraces variables such as temperature, pressure, composition, polymer molecular weight, polymer polydispersity, and isotopic label either in the solvent, or in the polymer, or in both [10,11]. In this work, we have studied five distinct polymeric systems (see Table 1) in $\mathrm{H}_{2} \mathrm{O}$ and/or in $\mathrm{D}_{2} \mathrm{O}$.

\subsection{System (PNIPAAM+ $\left.\mathrm{H}_{2} \mathrm{O}\right)$}

The effects of pressure and polymer composition (expressed as polymer wt.\%) on both the cp and material sp temperatures $\left(T_{\mathrm{cp}}\right.$ and $T_{\mathrm{sp}}$, respectively) are reported in Table 2 for (PNIPAAM $\left(\bar{M}_{\mathrm{w}}=\right.$ $615,500)+$ water). Isobars of cp's were obtained from the value of temperature where the intensity of transmitted light and scattered light suffer a sudden decrease and increase, respectively. Fig. 1 depicts the $T$ (wt.\% of polymer) phase diagram at a nominal pressure of $0.1 \mathrm{MPa}$, while Fig. 2 represents the pressure dependence of $\mathrm{cp}$ temperatures at a nominal composition wt. $\%$ of polymer $=4$. It should be emphasized that while the composition dependence of cp temperatures is fairly small in the range $0-20 \%$,

Table 2

Cloud-point temperatures, $T_{\mathrm{cp}}$, and spinodal temperatures, $T_{\mathrm{sp}}$, at distinct polymer wt. $\%, W_{\mathrm{p}}=\mathrm{wt} . \%$ of polymer, and pressure, $P$, for sample P1 (see Table 1 and text)

\begin{tabular}{rlll}
\hline$W_{\mathrm{p}}$ & $P(\mathrm{MPa})$ & $T_{\mathrm{cp}}(\mathrm{K})$ & $T_{\mathrm{sp}}(\mathrm{K})$ \\
\hline 1.030 & 0.10 & 307.20 & 321.6 \\
2.011 & 0.10 & 306.74 & 312.1 \\
2.502 & 0.10 & 306.65 & 309.2 \\
3.574 & 0.10 & 306.46 & 305.59 \\
3.701 & 0.10 & 305.50 & 308.5 \\
4.167 & 0.10 & 305.60 & 309.0 \\
4.167 & 1.00 & 305.70 & 308.2 \\
4.167 & 2.50 & 305.73 & 307.6 \\
4.167 & 3.00 & 305.86 & 307.4 \\
4.167 & 4.00 & 305.90 & \\
4.167 & 5.00 & 308.12 & 308.27 \\
4.167 & 20.0 & 308.32 & 310.7 \\
4.167 & 30.0 & 306.32 & 308.9 \\
4.167 & 40.0 & 306.10 & 307.8 \\
4.529 & 0.10 & 306.25 & 308.7 \\
5.646 & 0.10 & 306.40 & 309.1 \\
6.402 & 0.10 & 306.35 & 308.7 \\
6.774 & 0.10 & 306.46 & 309.1 \\
7.670 & 0.10 & 305.89 & 308.7 \\
8.839 & 0.10 & 306.20 & 310.9 \\
10.57 & 0.10 & 305.84 & 305.72 \\
11.46 & 0.10 & & \\
14.74 & 0.10 & 0.10 & \\
17.57 & & & \\
\hline
\end{tabular}




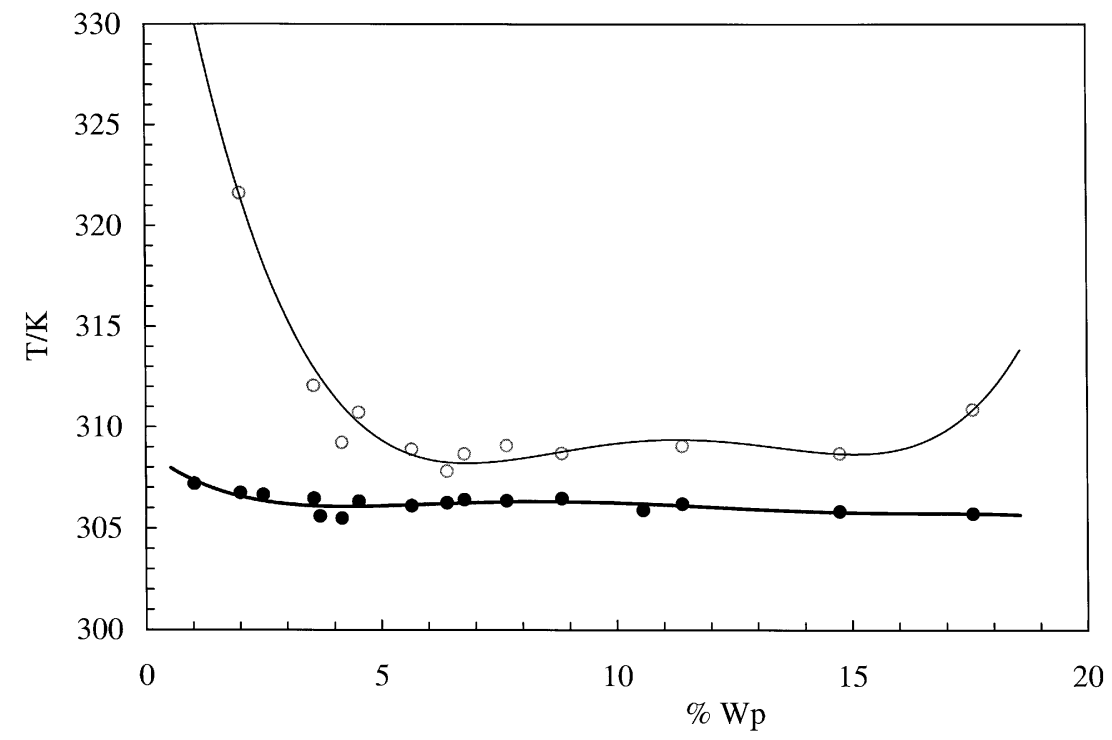

Fig. 1. Cloud-point and spinodal temperatures vs. polymer composition ( $\% W_{\mathrm{p}}=$ wt. $\%$ of polymer) for PNIPAAM $+\mathrm{H}_{2} \mathrm{O}$ (see Table 1) at a nominal pressure of $0.1 \mathrm{MPa}$. Thick line and $(\mathbf{O})$ represent experimental cp curve and individual cp temperatures, respectively. Thin line and $(\bigcirc)$ represent sp curve and individual sp temperatures, respectively.

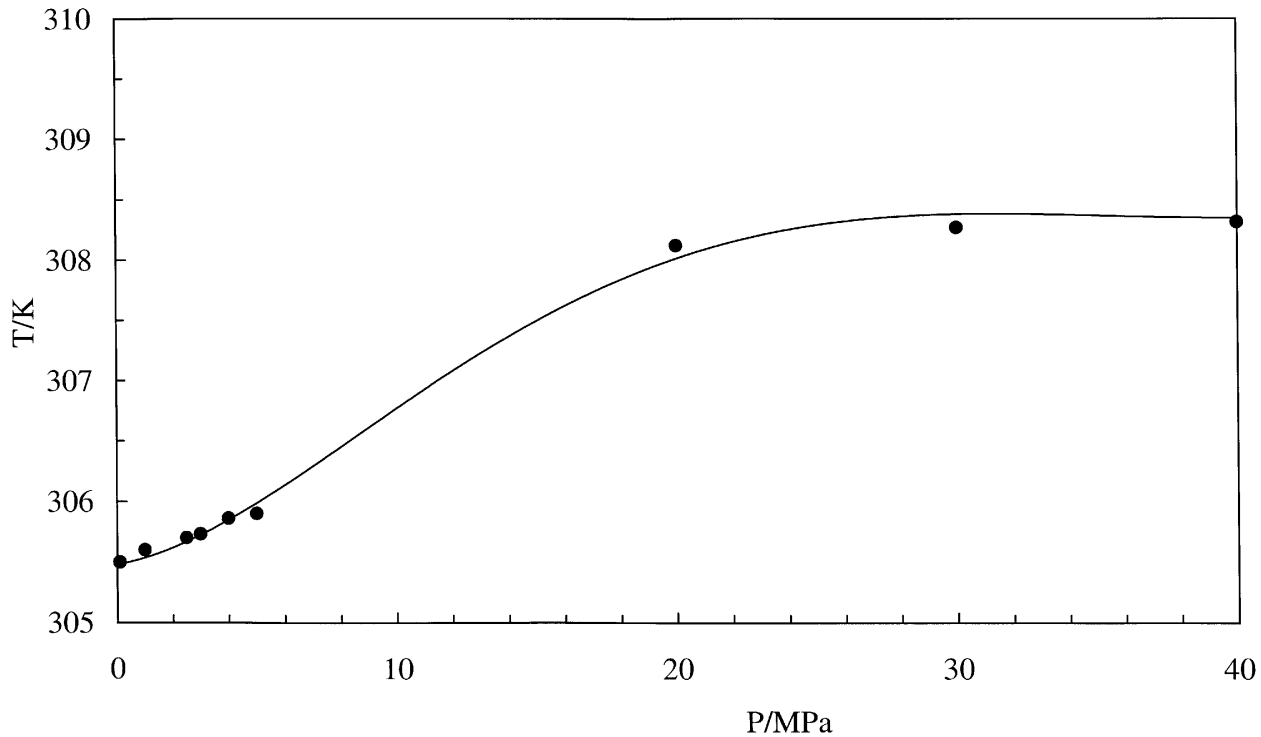

Fig. 2. Pressure effects on the cloud-point temperature of a nominal $4 \mathrm{wt} \%$ of polymer solution of $\left(\mathrm{PNIPAAM}+\mathrm{H}_{2} \mathrm{O}\right)$. Quantitative similar curves have been observed for higher and lower concentrations. 
in contrast, sp temperatures show a significant composition dependence. At temperatures lower than $\mathrm{cp}$ the system is in the one-phase homogeneous region. At temperatures between $\mathrm{cp}$ and $\mathrm{sp}$ the system is materially metastable with respect to phase separation, and formation of the second phase should occur by a nucleation-and-growth mechanism. Finally, at temperatures above the sp curve, the system is materially unstable and phase separation should occur by the so-called sp decomposition. In summary, one can state that under stable equilibrium conditions precipitation of the polymer-rich phase upon heating is almost independent of polymer concentration, but if one is able to avoid stable equilibrium separation by diving into the metastable region, then, polymer concentration should be taken into consideration. For (highly) polydisperse systems, the liquid-liquid critical point does not necessarily coincide with the minimum of the LCST curve, but more precisely with the point of tangency between the cp and the thermodynamic sp of material instability curves [11]. On the one hand, and rigorously speaking, operational and thermodynamic sp's may differ slightly [10]. On the other hand, our operational sp's could have only be determined within $\pm 1 \mathrm{~K}$. With these caveats in mind, analysis of our data indicate that the composition of the critical point at nominal $0.1 \mathrm{MPa}$ is wt. $\%$ of polymer $=6.5 \pm 0.5$. It is interesting to note that while cp temperatures increase as pressure increases (see Fig. 2), the opposite is observed for sp temperatures (Table 2). Since this comparison is being made at $4 \mathrm{wt} . \%$ (a value which is slightly smaller than that corresponding to critical composition), this unusual behavior seems to be an indication that critical composition is moving to lower values upon pressurization. It should also be noted that the slope of the temperature-pressure curve of phase separation (Fig. 2) decreases to zero as pressure increases to 400 bar. Using thermodynamic arguments, it can be shown [12] that regions of the phase diagram where the temperature of phase separation is pressure independent correspond to ideal mixing (null value for the excess volume, $v^{\mathrm{E}}$ ). On the other hand, for a LCST type of phase separation, a positive slope, as that observed in the major portion of Fig. 2 implies contraction $\left(v^{\mathrm{E}}<0\right)$ upon mixing, which favors miscibility as pressure is increasing.

Due to the fact that synthesis and characterization of PNIPAAM are difficult and tedious, and, above all, since other authors [3,5] have shown that temperature of transition is practically independent of polymer molecular weight, we have not considered the study of other molecular weights.

\subsection{System (copoly $\left.(P N I P A A M / V S)+\mathrm{H}_{2} \mathrm{O}\right)$}

Most of the copolymers of PNIPAAM studied so far [1] have been of the hydrophobically-modified type, which leads to a decrease of the LCST temperature. A similar effect is obtained when a hydrophilic compound is added to the solution [4] (competition between the compound and the polymer for dissolution), but the opposite should be observed (rise of the LCS temperature) if that compound is incorporated in the polymer chain. Therefore, if one seeks to use PNIPAAM copolymers as switches working at about human body temperature, it becomes necessary to incorporate biologically compatible compounds in the polymer chain in order to obtain slightly higher LCS temperatures.

In Fig. 3, plots of cp temperatures of several copolymers of PNIPAAM (Table 1) with a VS monomer, $\operatorname{copoly}(N$-isopropylacrylamide/1-deoxy-1-methacrylamide-D-glucitol), are represented. For purposes of comparison, PNIPAAM is also shown. The most striking feature is the abrupt increase in LCST observed for the lowest molecular weight copolymer (CP4, Table 1). This point was double-checked several times using fresh solutions of about 4.0 copolymer wt.\% each time. This observation, persi, indicates the proximity of a hypercritical point [10-14] (or double critical temperature point). This conclusion is corroborated by two other observations: (a) only in the close vicinity (3-5\%) of this concentration a 


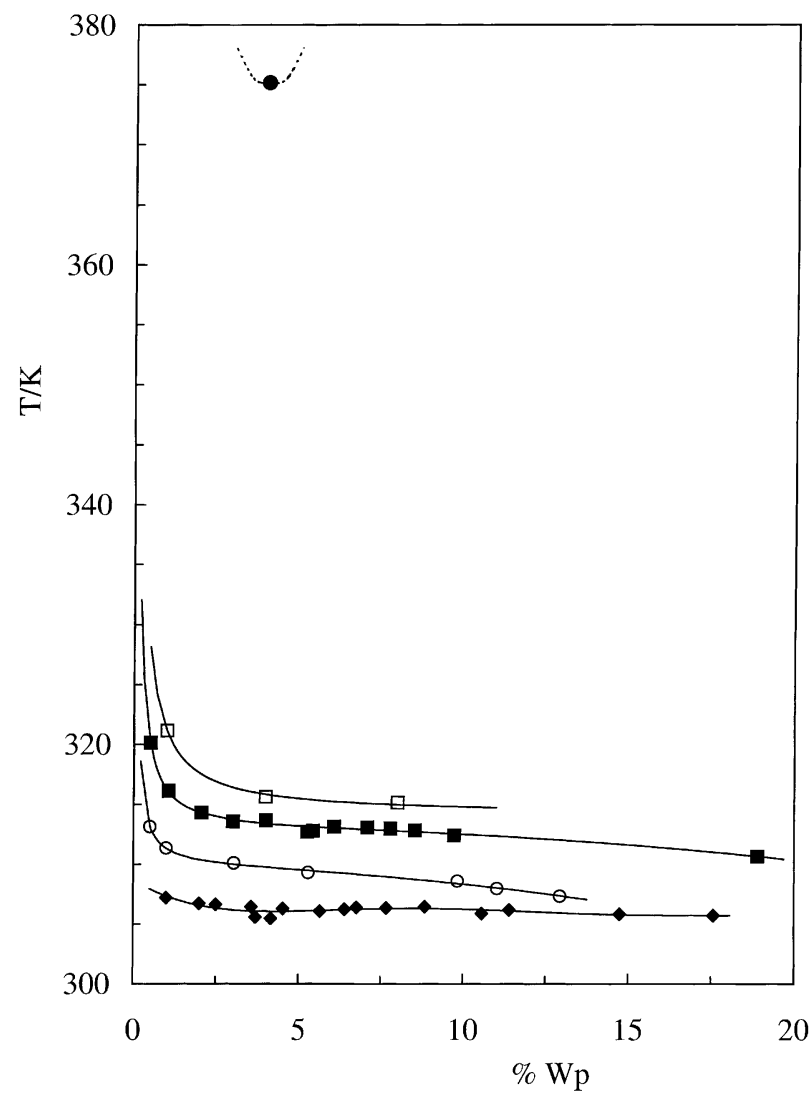

Fig. 3. Comparison between LCST's of PNIPAAM and copolymers in $\mathrm{H}_{2} \mathrm{O}$ at a nominal pressure of $0.1 \mathrm{MPa}$. $(\diamond) \mathrm{P} 1 ;(\mathrm{O}) \mathrm{CP} 1$;

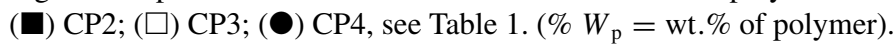

transition is observed - for higher and lower values a clear homogeneous one-phase is always obtained up to $440 \mathrm{~K}$; (b) deuterium substitution has a drastic effect on the LCST (see below). In other simpler words, the 13-14 mol\% copolymer of VS in PNIPAAM becomes completely soluble in $\mathrm{H}_{2} \mathrm{O}$, independently of temperature, for a weight average molecular weight smaller than 50,000 amu. In contrast with PNIPAAM [1,3], the copolymer LCST is molecular weight sensitive, becoming extremely molecular weight dependent at low $M_{\mathrm{w}}$ values.

Pressure effects have been investigated for two of the copolymers (CP1 and CP3) and are represented in Fig. 4. Results are qualitatively similar to those observed with PNIPAAM (Fig. 2). Present technical difficulties in using the high pressure cell at high temperatures in a silicon oil bath prevented us from obtaining results for $\mathrm{CP} 4$ at distinct pressures, which we plan to do in the near future. Contrary to the plotted curves (Fig. 4), pressure effects on LCST ought to be very significant here, and this should be explored in detail. As expected, a comparison of the two represented curves shows a slight increase in slope as molecular weight decreases. At temperature hypercritical conditions, the theoretical value for $\mathrm{d} T_{\mathrm{c}} / \mathrm{d} P$ of the transition is infinity [12]. 


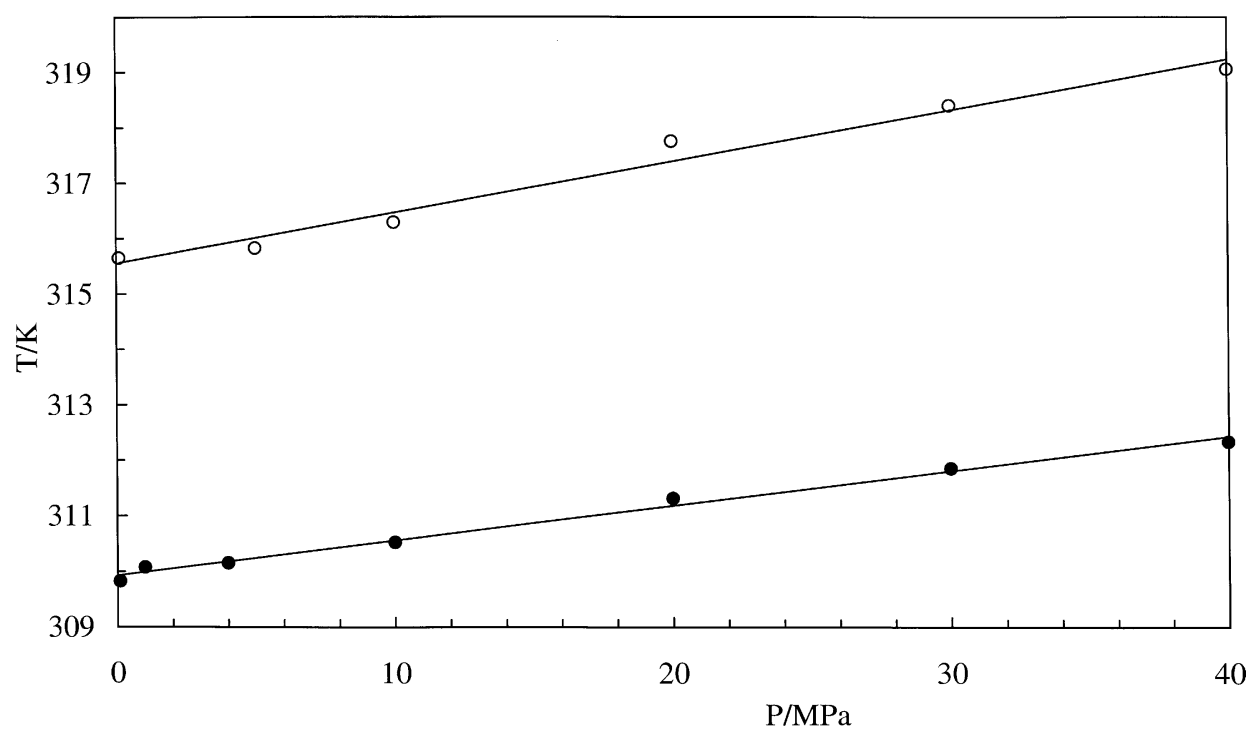

Fig. 4. Pressure effects on the cloud-point temperature of nominal $4 \mathrm{wt} \%$ of polymer solutions of copoly $\left(\mathrm{PNIPAAM} / \mathrm{VS}+\mathrm{H}_{2} \mathrm{O}\right)$. (O) $\mathrm{CP} 1 ;(\mathrm{O}) \mathrm{CP} 3$. Straight lines represent least-square fits to a first order polynomial.

We also studied the effect of physical addition of the VS to the solution of (PNIPAAM + water). A ternary mixture of PNIPAAM ( $83 \mathrm{wt} . \%)$ and VS (17wt.\%) of overall composition of $4.6 \mathrm{wt} . \%$ of polymer in water was prepared. As expected, the visual observation of the cp temperature dropped to $301.0 \pm 0.1 \mathrm{~K}$, as compared with identically concentrated solutions of PNIPAAM+water $(305.50 \pm 0.01 \mathrm{~K})$ and $\mathrm{CP} 1+$ water $(310.11 \pm 0.01 \mathrm{~K})$.

\subsection{Isotope effects}

Deuterium substitution in the solvent $\left(\mathrm{D}_{2} \mathrm{O}\right)$ for both $4 \mathrm{wt} . \%$ solutions of PNIPAAM and all copoly(PNIPAAM/VS) has shown a very small effect of ca. $1.0-1.2^{\circ} \mathrm{C}$ increase in the LCST compared to $\mathrm{H}_{2} \mathrm{O}$ with the single exception of the very low molecular weight copolymer (CP4). Here, as expected, the effect is drastic and we have observed a decrease of $56^{\circ} \mathrm{C}\left(T_{\text {LCST }}=319.05 \mathrm{~K}\right)$. In the case of PNIPAAM, Schild [1] has also reported (citing unpublished work) an elevation of $1.0 \pm 0.1^{\circ} \mathrm{C}$ for the LCST of aqueous PNIPAAM in $90 \% \mathrm{D}_{2} \mathrm{O}$. Fig. 5 shows the combined molecular weight and isotope effects for all copolymers, where, in the case of $\mathrm{H}_{2} \mathrm{O}$, we have assumed the Imre-Van Hook representation [15] of data. These authors developed a mean field scaling relation (Eq. (1)) where the expansion is done around an assumed hypercritical point, defined by $T_{\mathrm{c}}^{*}$ and $X^{*}\left(X^{*}=M_{\mathrm{w}}^{*-1 / 2}\right) . M_{\mathrm{w}}^{*}$ is the polymer molecular weight which locates the hypercritical point.

$$
\left|\frac{T_{\mathrm{c}}-T_{\mathrm{c}}^{*}}{T_{\mathrm{c}}^{*}}\right|=A\left|\frac{X-X^{*}}{X^{*}}\right|^{\alpha}, \quad X=M_{\mathrm{w}}^{-1 / 2} \text { for } \quad M_{\mathrm{w}}^{-1 / 2}>0
$$

This leads to the prediction of a temperature hypercritical point and, thus, to the existence of a UCST branch at high temperatures. The speculation is that the phase diagram of the copolymers + water is 


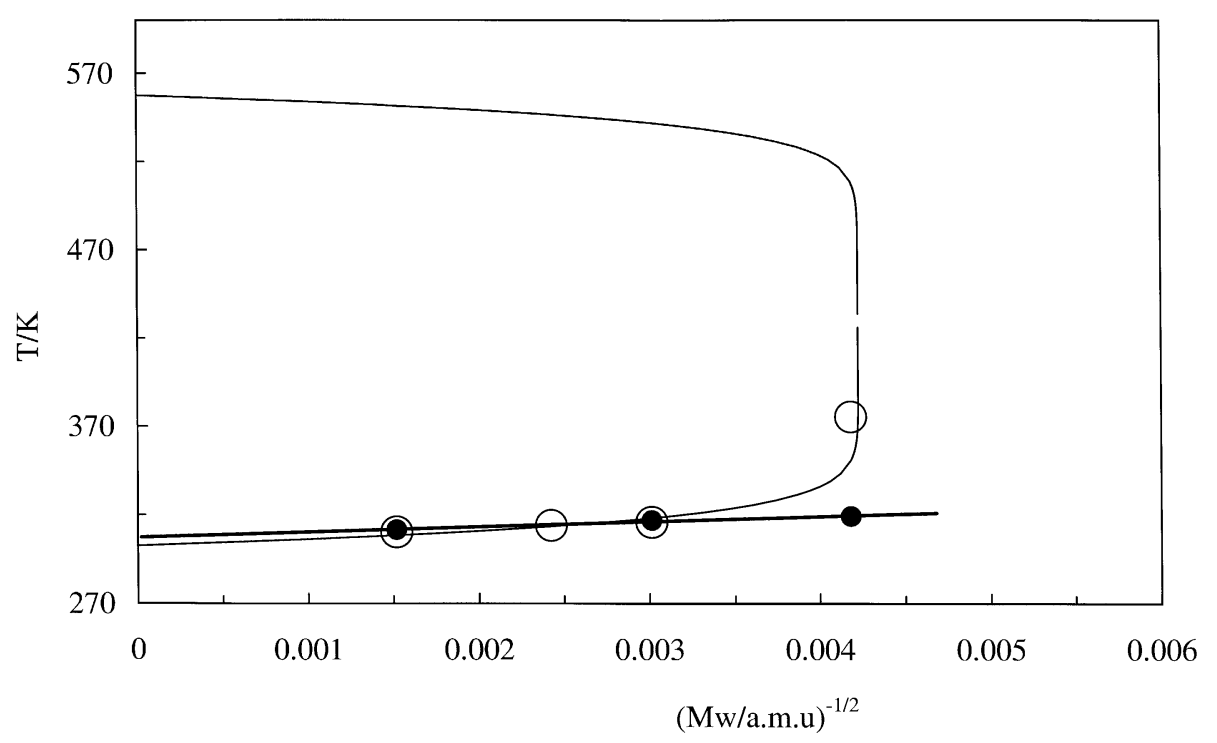

Fig. 5. Temperature of transition vs. $\left(1 / M_{\mathrm{w}}\right)^{1 / 2}$ for copolymers at 4 wt. $\%$ of polymer solutions in $\mathrm{D}_{2} \mathrm{O}(\boldsymbol{O})$ and in $\mathrm{H}_{2} \mathrm{O}(\mathrm{O})$ and at a nominal pressure of $0.1 \mathrm{MPa}$. Thick line is a first order least-squares fit to the deuterated solution. Thin line represents Eq. (1) for the hydrogenated solution fitted at a reference hypercritical point of $\left(T^{*}=430 \mathrm{~K} ; M_{\mathrm{w}}^{*}=0.559 \times 10^{5}\right)$. The two-phase region is inside the envelope.

inherently of the closed-loop type, though only the low temperature LCST branch is experimentally accessible. In accord with the predictions shown in Fig. $5\left(\mathrm{CP} 4+\mathrm{H}_{2} \mathrm{O}\right)$ was warmed up to $440 \mathrm{~K}$ with no observation of any reappearing homogeneous phase. One would have to go to much higher temperatures, but, there, thermal degradation of the copolymer would be inevitable.

Whether $\mathrm{D}_{2} \mathrm{O}$ also shows a temperature hypercritical point or not, it remains an open question, which only can be solved by performing measurements with copolymers of $M_{\mathrm{w}}<50,000$.

A final note of caution must be included. Both PNIPAAM and its copolymers have potential isotopic exchangeable hydrogens. These are those bonded to nitrogen (PNIPAAM and copolymers) and oxygen (copolymers). In the case of $4 \mathrm{wt} . \%$ of polymer solutions, we have estimated that the number (moles) of potentially exchangeable $\mathrm{H}$ (in $-\mathrm{OH}$ groups) in copolymers per mol of $\mathrm{D}_{2} \mathrm{O}$ is about $4 \times 10^{-3}$. This ratio shifts a probable isotopic exchange reaction towards the formation of a partially deuterated $(D / H=6 / 11)$ copolymer, maintaining the solvent at roughly the original content of deuterium. Similar conclusions are reached for PNIPAAM (in-NH groups) where that ratio is about $7 \times 10^{-3}$ with the formation of a partially deuterated $(\mathrm{D} / \mathrm{H}=1 / 10)$ polymer. Thus, assuming that the isotopic exchange reaction is not kinetically controlled, one is in fact studying systems of partially deuterated polymers in a deuterated solvent. This remark does not affect the previous conclusions though. In the case of copolymers the analysis becomes additionally complicated by the possibility of formation of multiple intramolecular hydrogen-bonds.

\section{Acknowledgements}

RGA and JS are grateful to PRAXIS (BD/17179/98 and BCC/16424/98, respectively) for financial support. Research at ITQB was supported by the Praxis Program (no. 2/2.1/Qui/178/94). 


\section{References}

[1] H.G. Shild, Prog. Polym. Sci. 17 (1992) 163-249.

[2] H.G. Shild, M. Muthukumar, D.A. Tirrel, Macromolecules 24 (1991) 948-952.

[3] H.G. Shild, D.A. Tirrel, J. Phys. Chem. 94 (1990) 4352-4356.

[4] Y.-H. Kim, I.C. Kwon, Y.H. Bae, S.W. Kim, Macromolecules 28 (1995) 939-944.

[5] S. Fujishige, K. Kubota, I. Ando, J. Phys. Chem. 93 (1989) 3311-3313.

[6] J. Klein, D. Herzog, Makromol. Chem. 188 (1987) 1217-1232.

[7] H.C. de Sousa, L.P.N. Rebelo, J. Chem. Thermodyn. 32 (2000) 355-387.

[8] J. Szydlowski, L.P.N. Rebelo, W.A. Van Hook, Rev. Sci. Instrum. 63 (1992) 1717-1725.

[9] F. Kiepen, W. Borchard, Macromolecules 21 (1988) 1784-1790.

[10] M. Luszczyk, L.P.N. Rebelo, W.A. Van Hook, Macromolecules 28 (1995) 745-767.

[11] H.C. de Sousa, L.P.N. Rebelo, J. Polym. Sci. Polym. Phys. 38 (2000) 632-651.

[12] L.P.N. Rebelo, Phys. Chem. Chem. Phys. 1 (1999) 4277-4286.

[13] L.P.N. Rebelo, W.A. Van Hook, J. Polym. Sci. Polym. Phys. 31 (1993) 895-897.

[14] L.P.N. Rebelo, H.C. de Sousa, W.A. Van Hook, J. Polym. Sci. Polym. Phys. 35 (1997) 631-637.

[15] A. Imre, W.A. Van Hook, J. Phys. Chem. Ref. Data 25 (1996) 637-661. 